\title{
Experimental Study on Pulse Detonation Engine with Two-Phase Inhomogeneous Mixture
}

\author{
Jishuang Gong ${ }^{1}$ and $\mathrm{Hu} \mathrm{Ma}^{2}$ \\ ${ }^{1}$ School of Aeronautics and Astronautics, Sun Yat-sen University, Guangzhou, 510275, China \\ ${ }^{2}$ School of Mechanical Engineering, Nanjing University of Science and Technology, Nanjing 210094, China \\ Correspondence should be addressed to Jishuang Gong; gongjsh@mail.sysu.edu.cn
}

Received 3 April 2020; Revised 19 July 2020; Accepted 5 August 2020; Published 1 September 2020

Academic Editor: Qiaofeng Xie

Copyright (C) 2020 Jishuang Gong and Hu Ma. This is an open access article distributed under the Creative Commons Attribution License, which permits unrestricted use, distribution, and reproduction in any medium, provided the original work is properly cited.

\begin{abstract}
In order to investigate the effects of fuel distribution on the operation of two-phase pulse detonation engine (PDE), a series of cold flow and multicycle PDE experiments was carried out with 9 mixing schemes. Homogeneity degree with fuel distribution considered in terms of space and time was proposed to quantitatively evaluate the mixing of liquid fuel and air by particle image velocimetry (PIV) in cold flow experiments. Operation stability of multicycle PDE was presented by statistical analysis of peak pressure at the outlet of a detonation tube. The relationship between operation stability and homogeneity degree was quantitatively elaborated. These experimental results indicated that not only using mixing reinforcement devices (such as pore plate and reed valve) was fuel distribution improved but also the effect of inlet ways on the homogeneity degree was weakened. The homogeneity degree of fuel distribution $\zeta=0.72$ was a critical value for stable working of multicycle PDE. When homogeneity degree was lower than 0.72 , stable state was not maintained and detonation wave in some cycles was not established due to poor fuel distribution. Therefore, it is necessary to hold homogeneity degree larger than 0.72 to achieve stable operation of PDE. These results contribute to enhancing the operation stability and offering guidelines for the design of PDE's mixing scheme.
\end{abstract}

\section{Introduction}

Pulsed detonation engine (PDE) obtains thrust by intermittent detonation wave. Numerous theoretical, experimental, and numerical studies were carried out since PDE poses higher thermodynamic efficiency [1]. Gaseous fuels will be more easily applied to PDE because of the easier mixing with oxidants [2-5], but their energy density is lower than that of liquid fuel; then, the application to PDEs is not that promising. Liquid fuel has now been the focus in this field $[6,7]$. Studies on spray detonation presented that PDE efficiency (check again!) is highly related to the droplet size and vaporization of liquid fuel $[8,9]$; thus, atomization and vaporization of liquid fuel should be considered for liquidfueled PDE.

Cheatham et al. conducted single-cycle performance estimations of an idealized liquid-fueled PDE by numerically simulating the detonation of JP-10 fuel droplets in oxygen and in air [10]. Their results suggested that for small enough droplets or with sufficient prevaporization of the fuel, liquidfueled PDE will provide comparably single-cycle propulsive performance to gaseous-fueled PDE. However, the performance would decrease when droplet sizes were too large that a self-propagating detonation wave cannot be obtained at the end of the tube. By comparing simulation results to experimentally observed trends, the conclusions were drawn that smaller droplet sizes and higher levels of heating and prevaporization are likely to increase the ease of detonation initiation of liquid-fuelled mixtures [11].

Simulations of a single ideal-tube PDE fueled with multiphase $\mathrm{JP} 10-\mathrm{O}_{2}$ and JP10-air mixtures were reported by Tangirala et al. [12]. For the diameter ranges of the droplets $\left(3 \mu \mathrm{m}-10 \mu \mathrm{m}\right.$ for fuel-air and $10 \mu \mathrm{m}-20 \mu \mathrm{m}$ for fuel- $\mathrm{O}_{2}$ mixtures) and the equivalence ratio considered in their investigations, the predicted velocity defect was $5 \%$ of the quasisteady detonation velocity through gas-phase mixtures of 


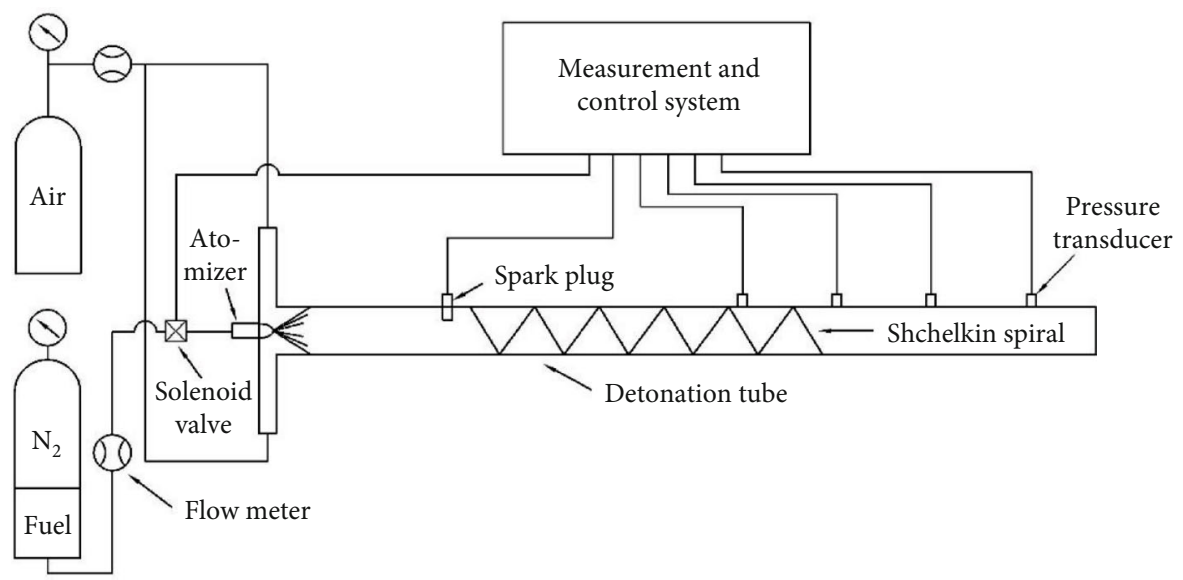

FIgURE 1: Schematic of PDE experimental setup.

JP10- $\mathrm{O}_{2}$ /air, and specific impulse $I_{\text {spf }}$ of the PDE initially fueled with gaseous fuel was higher (1-5\%) than the $I_{\text {spf }}$ of a PDE initially fueled with a multiphase JP10- $\mathrm{O}_{2} /$ air mixture.

It has been demonstrated in these studies that improving initial atomization and vaporization levels of liquid fuel could provide a benefit to the performance of PDE. The atomization with a high-speed coaxial airflow was discussed by Lasheras et al. [13]. They stated that the high-speed airflow driven by stagnation pressures is required to atomize the injected fuel. Sauter mean diameter (SMD) values below $10 \mu \mathrm{m}$ can be achieved when gas-injection velocities were greater than $220 \mathrm{~m} / \mathrm{s}$. Wang et al. [14] investigated the influences of atomization on PDE by employing laser light scattering for the measurements of mean droplet size. It was observed that equivalence ratio limits turned wide and detonation wave velocity increased as the gasoline droplet size decreased.

Tucker et al. reduced the evaporation time of liquid fuel in a pulsed detonation engine through a fuel flash vaporization system $[15,16]$. The results showed that the flash vaporization system quickly provides a detonable mixture for all of the fuels tested without coking the fuel lines, and ignition time has nearly no dependence on fuel injection temperatures. In their works, the successful detonation of flash vaporized JP-8 in air was achieved over a range of fuel temperatures and fuel-to-air ratios.

Miser et al. [17] built a concentric tube heat exchanger using the waste heat generated by a PDE to produce a flash vaporization of a JP-8/air mixture. The duration of the steady-state tests exceeded the operating time of any previous JP-8-fueled PDEs, which is higher than twenty minutes and limited only by fuel storage capacity.

Helfrich et al. [18] studied the effect of fuel temperature on PDE's performance with different liquid fuels by the concentric tube heat exchanger. In their works, for all fuels except JP-10, increasing the fuel injection temperature leads to the decrease of both DDT (deflagration-todetonation transition) time (by 15\%) and detonation distance (by up to $30 \%$ ) but causes the increase of detonation percentage by up to $180 \%$ and barely affects the ignition time.
Fan et al. [19] discussed the beneficial effects of the fuel pretreatments on PDRE performance with five concentriccounter-flow heat exchangers. The outcomes showed that with the aid of fuel preheating, the time and distance of DDT for liquid kerosene were remarkably reduced and the operation time was greatly prolonged. With the increase of fuel temperature, the specific impulse rises from $97.3 \mathrm{~s}$ at $25^{\circ} \mathrm{C}$ to $115.4 \mathrm{~s}$ at $200^{\circ} \mathrm{C}$.

Even fuel was vaporized from liquid to gas, the fuel distribution in detonation tube still largely influences PDE's performance, and this aspect is now attracting considerable attention from researchers [20, 21]. Tunable diode laser and absorption spectroscopy techniques had been applied to provide time-resolved fuel mass fraction measurements in PDE by Brophy et al. $[22,23]$. It was found that the fuel mass fraction distribution within a PDE inherently affects the overall system performance in terms of both the initiation characteristics and the resulting fuel-based-specific impulse performance values. A stratified axial fuel distribution, where a near-stoichiometric mixture occurs near the initiation end of a combustor and a leaner mixture appears near the combustor exit, has significant operation benefits, such as promoting rapid ignition/DDT and increasing fuel-based specific impulse compared to uniform fuel distribution mixtures with the same aggregate fuel mass fraction.

Perkins and Sung [24] analyzed detonation cycles of nonuniform $\mathrm{H}_{2}$-air mixtures using two-dimensional numerical simulation. The results presented that for an $\mathrm{H}_{2}$-air system, good fuel-air mixing is not a prerequisite for optimal detonation tube performance. In order to investigate spray detonation, droplet diameter distribution and two-phase mixture homogeneity were considered by Brett [25]. In his research, Mie scattering was used to image two-phase mixture and the mixture homogeneity was ascertained by statistical analysis of these images. It can be concluded that small changes in homogeneity might have little impact on the detonation wave velocity.

As mentioned above, there are many existing studies on the influence of mixture heterogeneity on PDE performance, but those results are mostly concerned with gas phase conditions. Two-phase PDE's performance was influenced not 


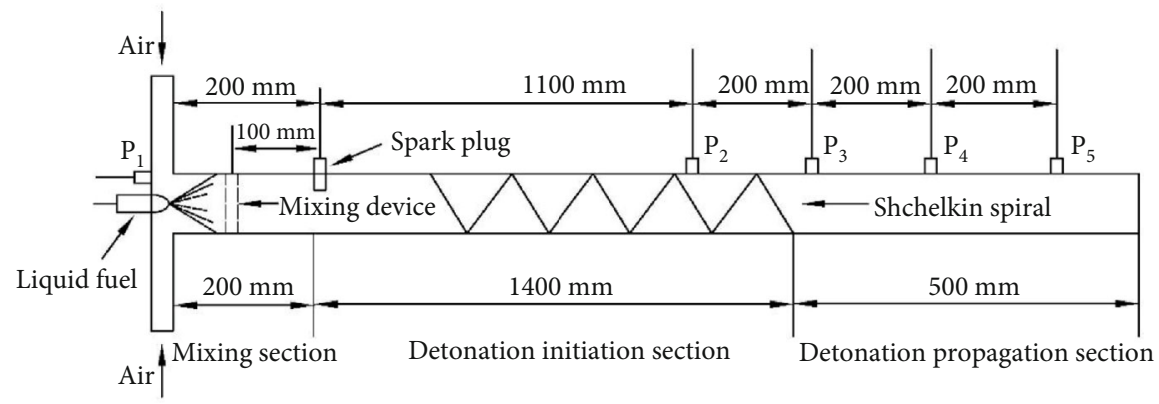

Figure 2: Detonation tube.

only by atomization and evaporation of liquid fuel but also by mixture homogeneity. This is a matter of great concern to practical PDE; however, there was little detailed research on this aspect. Therefore, a series of multicyclic two-phase PDE experiments was carried out with 9 mixing strategies to quantitatively investigate the effect of two-phase mixture homogeneity on two-phase PDE operation.

\section{Experimental Setup}

2.1. PDE Experimental Setup. PDE's experimental systems are set up to study multicyclic operation of two-phase airbreathing PDE. The experimental test rig is composed of a propellant supply system, a detonation tube, a measurement and control system, and an ignition system, as illustrated in Figure 1.

The propellant supply system consists of an oxidizer supply equipment and a fuel supply equipment. The fuel supply equipment provides liquid gasoline fuel (No. 97 in China) which contained $97 \% \mathrm{C}_{8} \mathrm{H}_{18}$ to the detonation tube by a pressure-swirl atomizer. The gasoline is stored in a cylinder, within which the upper part was full of nitrogen. The mass flow rate of gasoline is measured by a flow meter and is controlled by the pressure of nitrogen. Sauter mean diameter is measured by shadowgraph (71 $\mu \mathrm{m}$ in these experiments).

Air, as an oxidizer, is supplied to a detonation tube by an oxidizer supply equipment. A flow meter and a regulating valve are used to measure and control the mass flow rate of air. The solenoid valve in the fuel supply system is installed to ensure periodic detonations. The average equivalence ratio of 1.5 is fixed at all experiments.

The detonation tube is $50 \mathrm{~mm}$ in inner diameter and $2100 \mathrm{~mm}$ in length, which includes a mixing section of $200 \mathrm{~mm}$, a detonation initiation section of $1400 \mathrm{~mm}$, and a detonation wave propagation section of $500 \mathrm{~mm}$, as shown in Figure 2. Mixing devices such as a pore plate or a reed valve are set in the mixing section to get a different fuel distribution. More details about the mixing devices are shown in Figures 3 and 4. Detonable mixture is ignited in detonation initiation section by an ignition system. The ignition system adopts spark plug discharge with a frequency of $14 \mathrm{~Hz}$ and delivers energy of $1 \mathrm{~J}$. In order to decrease DDT run-up distance and time, a Shchelkin spiral with a blockage ratio of 0.422 is placed in the detonation initiation section. The length and outside diameter of Shchelkin spiral are

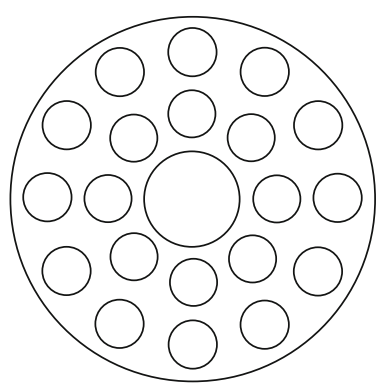

Figure 3: Pore plate.

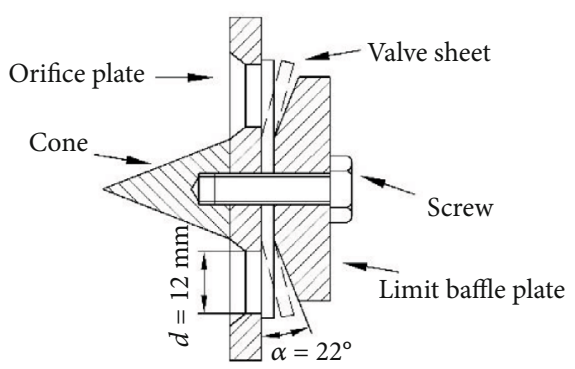

Figure 4: Reed valve.

$1300 \mathrm{~mm}$ and $50 \mathrm{~mm}$, respectively, which has a wire diameter of $6 \mathrm{~mm}$ and $35 \mathrm{~mm}$ distance between coils. The blockage ratio of 0.422 is close to the optimum value of 0.43 given by Peraldi et al. [26].

To record the pressure history along the detonation tube, dynamic piezoelectric pressure transducers are flushmounted in the detonation tube, as shown in Figure 2. Signals from these transducers are transmitted to a 6-channel simultaneous sampling module through a signal conditioner. The sampling frequency is $500 \mathrm{kHz}$ in all experiments.

2.2. PIV Experimental Setup. For the two-phase mixture, it is very difficult to achieve a uniform mixture and regions with more gas or more liquid are likely to appear. Therefore, the homogeneity of the mixture must be considered in twophase PDE. Particle image velocimetry (PIV) is employed to image the fuel distribution with time in the detonation tube, as shown in Figure 5, where an image analysis software, a high-speed CMOS camera, and a double-cavity Nd:YAG laser are implemented. A pulse laser sheet, which shoots from 


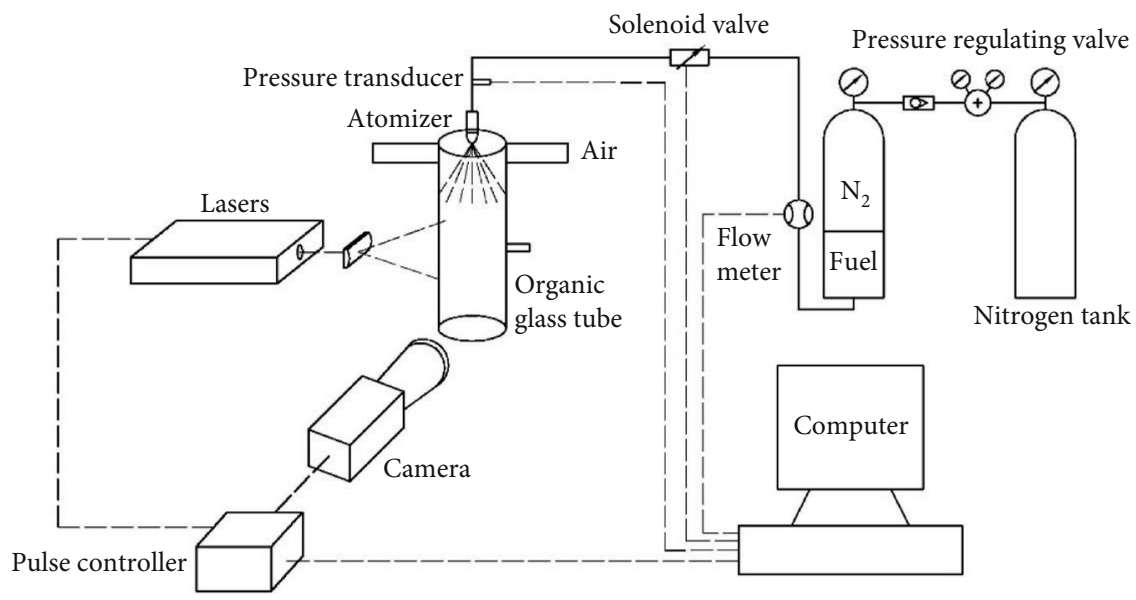

FIgURE 5: PIV experimental setup.

the double-cavity Nd:YAG laser with pulse energy of $30 \mathrm{~mJ}$ at $1 \mathrm{kHz}$, is arranged to illuminate the center plane near the spark plug. The high-speed CMOS camera is used to record particle distribution of the illuminated region with $1280 *$ 800 pixels and $1 \mathrm{kHz}$. An organic glass tube with $50 \mathrm{~mm}$ inner diameter and $2 \mathrm{~mm}$ thickness is incorporated as the observation window.

2.3. Mixing Schemes. To get a different fuel distribution, 9 mixing schemes, as shown in Table 1, are carried out in the mixing section for these experiments. Tangential, axial, and radial air inlet ways, as shown in Figures 6(a)-6(c), respectively, are adopted to provide air to the detonation tube by four inlet branches. Mixing reinforcement devices, such as a pore plate and a reed valve, are installed in the mixing section to achieve different fuel distribution and mixture homogeneities. The structure of the pore plate which has 0.54 blockage ratio is plotted in Figure 3. The diameter is $14 \mathrm{~mm}$ for big pore and $7 \mathrm{~mm}$ for small pores.

The reed valve is composed of an orifice plate, a cone, a valve sheet, a limit baffle plate, and a screw, as illustrated in Figure 4 . The left part of the reed valve is connected to the air inlet system and the right end goes to the detonation initiation section. The orifice plate has eight orifices which are evenly distributed along the circumference of the orifice plate. The diameter of orifices is $d=12 \mathrm{~mm}$. The valve switches on (as shown in dashed line of Figure 4) in the filling process of combustible mixture driven by the pressure difference between left and right of the valve, the maximum opening angle of the valve sheet is $\alpha=22^{\circ}$, and the blockage ratio of the reed valve is 0.54 .

\section{Two-Phase Mixture Homogeneity}

For PDE, whether the combustible mixture can be ignited or not depends largely on two-phase mixture homogeneity near the spark plug. Therefore, fuel distribution near spark plug with different mixing schemes is captured by PIV. Figure 7 (a) shows the fuel distribution near spark plug for the tangential air inlet way without a mixing reinforcement
TABle 1: Nine different mixing schemes.

\begin{tabular}{|c|c|c|c|}
\hline \multirow{2}{*}{$\begin{array}{l}\text { Air inlet } \\
\text { ways }\end{array}$} & \multirow{2}{*}{$\begin{array}{l}\text { No-mixing reinforcement } \\
\text { device }\end{array}$} & \multicolumn{2}{|c|}{$\begin{array}{l}\text { Mixing } \\
\text { reinforcement } \\
\text { device }\end{array}$} \\
\hline & & $\begin{array}{l}\text { Pore } \\
\text { plate }\end{array}$ & $\begin{array}{l}\text { Reed } \\
\text { valve }\end{array}$ \\
\hline Tangential & Case 1 & Case 4 & Case 7 \\
\hline Radial & Case 2 & Case 5 & Case 8 \\
\hline Axial & Case 3 & Case 6 & Case 9 \\
\hline
\end{tabular}

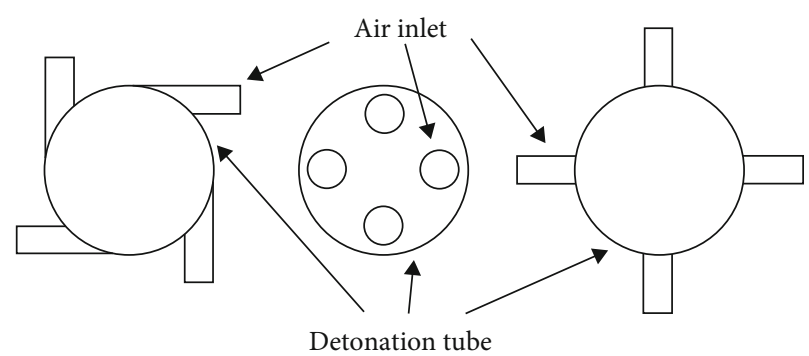

(a)

(b)

(c)

FIgURE 6: Tangential (a), axial (b), and radial (c) air inlet ways.

device. Centrifugal forces are generated by the tangential airflow in the tube. The centrifugal forces lead to a spiral movement of fuel droplets along the tube wall, and there are a little fuel droplets near the axis of the tube. It can be seen from Figure 7(b) that the pore plate reduces the centrifugal forces of air and improves the fuel distribution near the spark plug.

Figure 7 only gives instantaneous spatial distribution of liquid fuel. A deserving notice is that the two-phase mixture homogeneity not just relates to spatial distribution but to time as well. To further quantitative study mixture homogeneity for 9 mixing schemes, the space and time are considered by the statistical analysis of these images from PIV. Digital information with gray value of each pixel points is contained in these images. The homogeneity degree of fuel distribution, $\zeta$, can be calculated by following equation: 


$$
\zeta=1-\frac{S_{G}^{2}}{\bar{G}^{2}}=1-\frac{1 /((n-1) \times(m-1)) \sum_{(i, t)=(1,1)}^{(n, m)}\left(G_{i, t}-\bar{G}\right)^{2}}{\bar{G}^{2}},
$$

where $G_{i, t}$ is the gray value of pixel point $i$ at time $t, n$ is the sum of pixel points, and $m$ is the total number of pictures. We set $m=100, \bar{G}$ is the average gray value of pixel points for all pictures. The closer the homogeneity degree $\zeta$ is to 1 , the more homogeneous the liquid fuel distribution is.

Figure 8 shows homogeneity degree for nine mixing schemes. It can be seen from Figure 8 that the homogeneity degree changes from 0.32 to 0.87 for different mixing schemes. The air inlet ways have larger effects on the homogeneity degree without mixing reinforcement devices than those arrangements with the pore plate or reed valve. The homogeneity degree of axial inlet way is larger than that of tangential inlet way for all cases. In comparison with tangential inlet way, the homogeneity degree of axial inlet way has been increased by $103 \%$ for the test with no mixing reinforcement devices, but by $20.8 \%$ for the one with the pore plate and $4.88 \%$ for the reed valve incorporated case. The mixing reinforcement devices, such as the pore plate and reed valve, in addition to improve the homogeneity degree near spark plug, reduce the effect of inlet ways on the homogeneity degree as well and the reed valve outperforms the pore plate.

\section{Effect of Homogeneity Degree on PDE}

4.1. Multicycle PDE Experiments. To investigate the effect of mixing homogeneity on PDE operating characteristics, a series of multicycle two-phase detonation experiments is carried out with nine different mixing schemes. In these experiments, the filling velocity of air is about $30 \mathrm{~m} / \mathrm{s}$, the equivalence ratio of fuel/air mixture is 1.5 , the ignition frequency of spark plug was $14 \mathrm{~Hz}$, and the ambient pressure and temperature are $1 \mathrm{~atm}$ and $280 \mathrm{~K}$, respectively.

Figure 9(a) shows the pressure history of Case 1 where tangential inlet way is utilized and no mixing reinforcement device is employed. It can be seen from Figure 9(a) that the two-phase mixture is ignited only once during the period of $1 \mathrm{~s}$ with an ignition frequency of $14 \mathrm{~Hz}$ and the deflagration flame dose not translate to detonation wave. This is because centrifugal forces generating from rotating airflow cause a bad homogeneity degree, which becomes seriously fuel-rich near the spark plug and fuel-lean near the axis in the detonation tube. Its homogeneity degree is 0.32 for case 1 , making the ignition hard.

Figures 9(b) and 9(c) show the pressure history along the detonation tube for Case 2 and Case 3, respectively. Compared with Case 1, achieving successful ignition is significantly improved due to the improvement of the mixture homogeneity degree, but detonation wave is still not obtained in several cycles. This indicates that the homogeneity degrees for Case 2 and Case 3 still could not meet the requirement of $\mathrm{PDE}$, which means it is difficult to establish detonation wave even if ignition succeeds in some cycles.

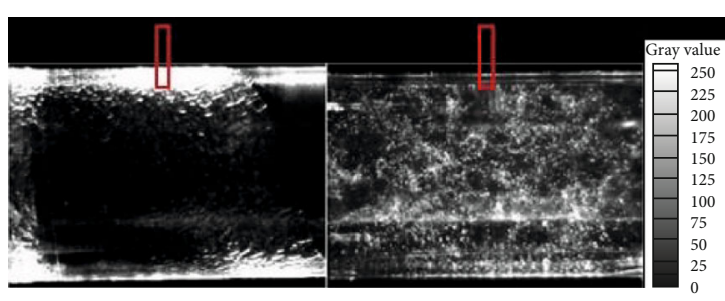

(a) Case 1

(b) Case 4

Figure 7: Fuel distribution recorded by PIV.

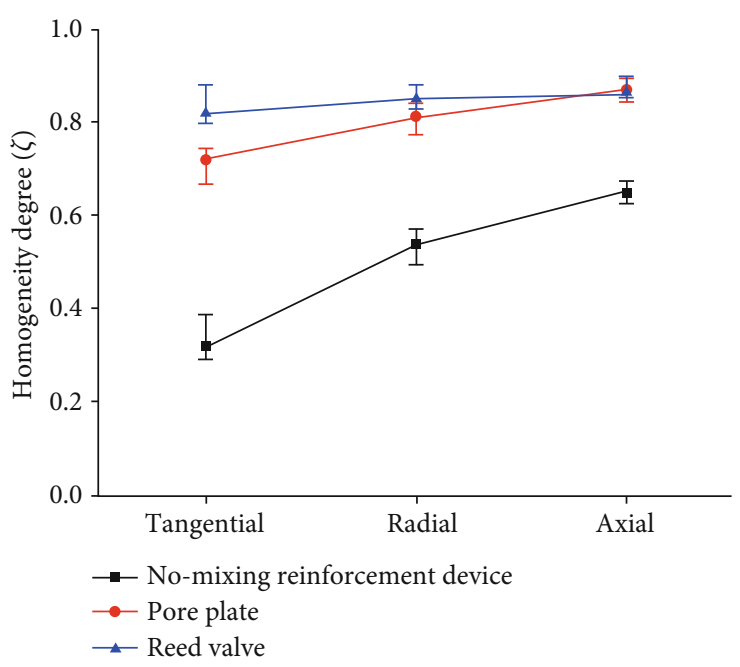

Figure 8: Homogeneity degree with 9 mixing schemes.

To improve fuel distribution and operation stability of PDE, the pore plate is installed $100 \mathrm{~mm}$ upstream to the spark plug in Case 4, Case 5, and Case 6.

Figure 10(a) shows the pressure history for Case 4 . It can be seen that the detonation wave is established under all states except one throughout the period of $1 \mathrm{~s}$ with an ignition frequency of $14 \mathrm{~Hz}$. It is found that the pore plate is able to reduce the negative impact of centrifugal forces from rotating airflow on fuel distribution. Compared with that for Case 1, the operation stability of PDE for Case 4 significantly gets enhanced as the pore plate improves the homogeneity degree of fuel-air mixture from 0.32 for Case 1 to 0.72 for Case 4.

Figures 10(b) and 10(c) show the pressure histories for Case 5 and Case 6, respectively. It can be seen from these figures that successful detonation wave initiation is always reached for Case 5 and Case 6. These indicate that the homogeneity degrees for Case 5 and Case 6 can satisfy the requirement of PDE. Compared with Figure 9, the pore plate can improve operation stability of PDE for all different air inlet ways.

Figure 11 shows the pressure history along the detonation tube with the reed valve implemented. As shown in Figure 11, PDE with the reed valve can operate more steadily for different air inlet ways. It indicates that besides improving the homogeneity to meet the requirements of PDE, the reed valve also reduces the effect of air inlet ways on PDE. 


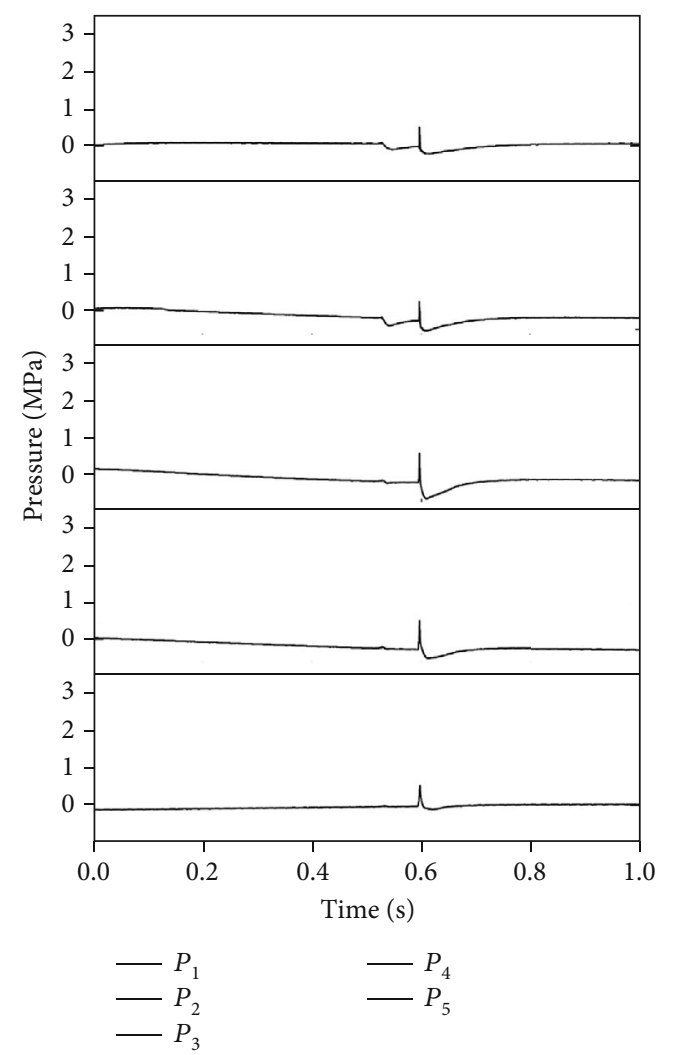

(a) Case 1

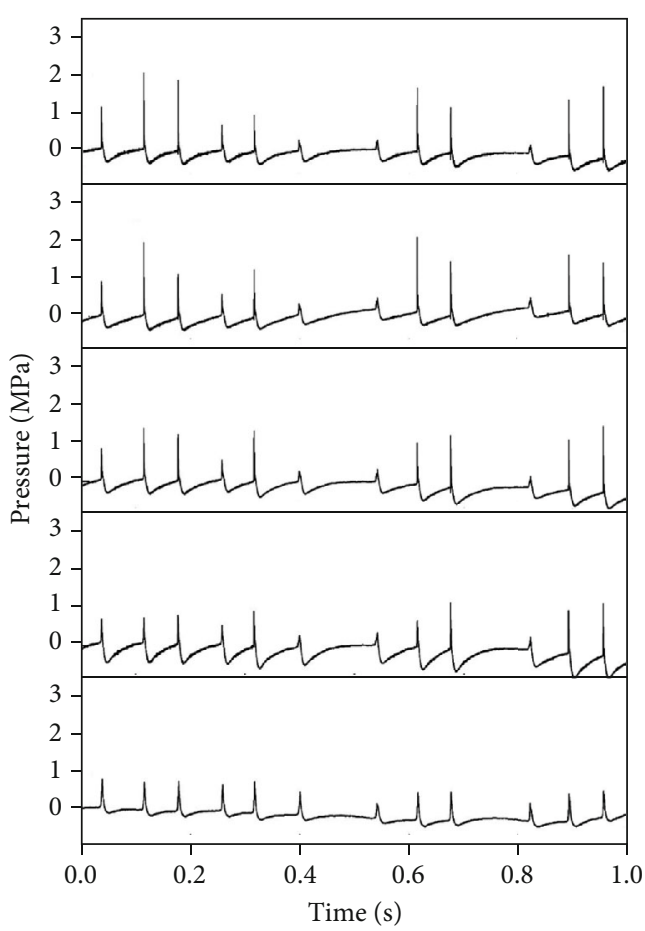

$$
\begin{array}{ll}
-P_{1} & -P_{4} \\
-P_{2} & -P_{5} \\
P_{3} &
\end{array}
$$

(b) Case 2

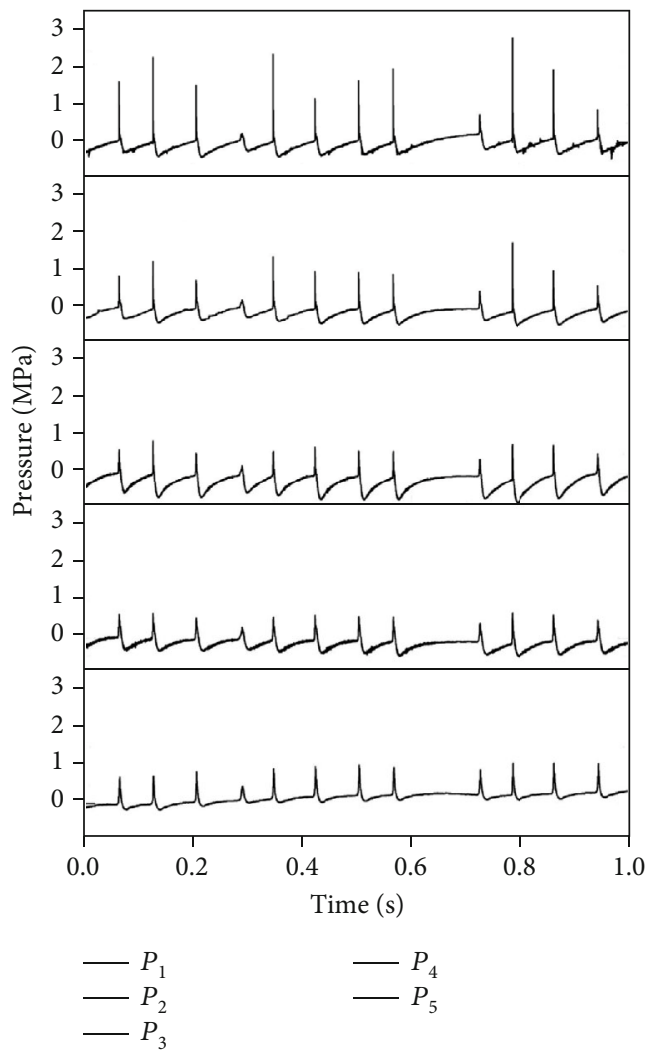

(c) Case 3

FIGURE 9: Pressure history for no-mixing reinforcement device. 


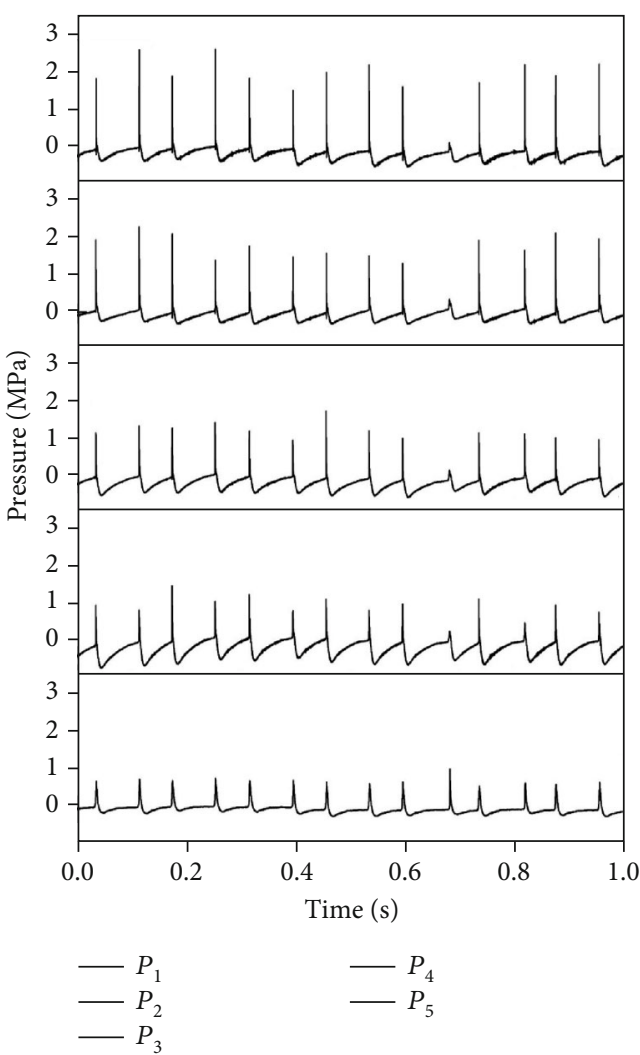

(a) Case 4
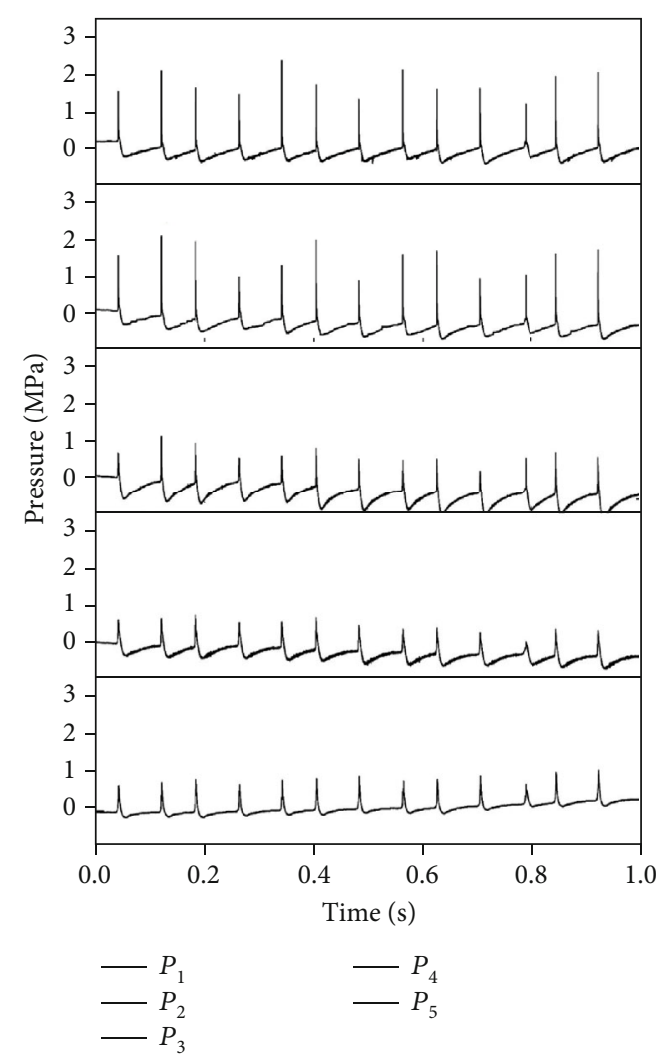

(b) Case 5

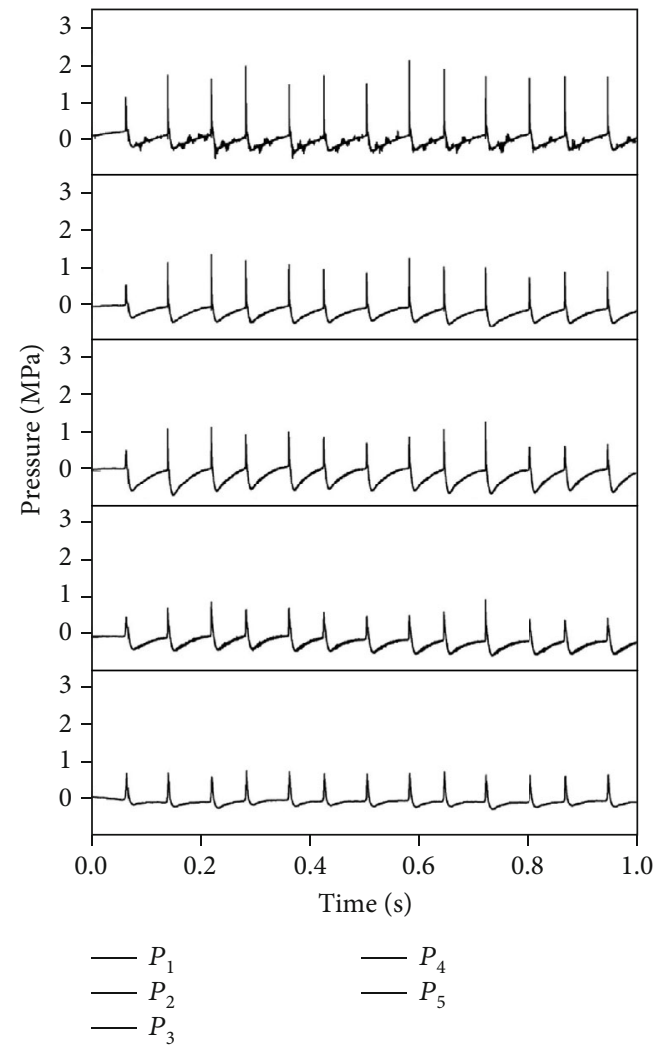

(c) Case 6

FIgURE 10: Pressure history for the pore plate. 


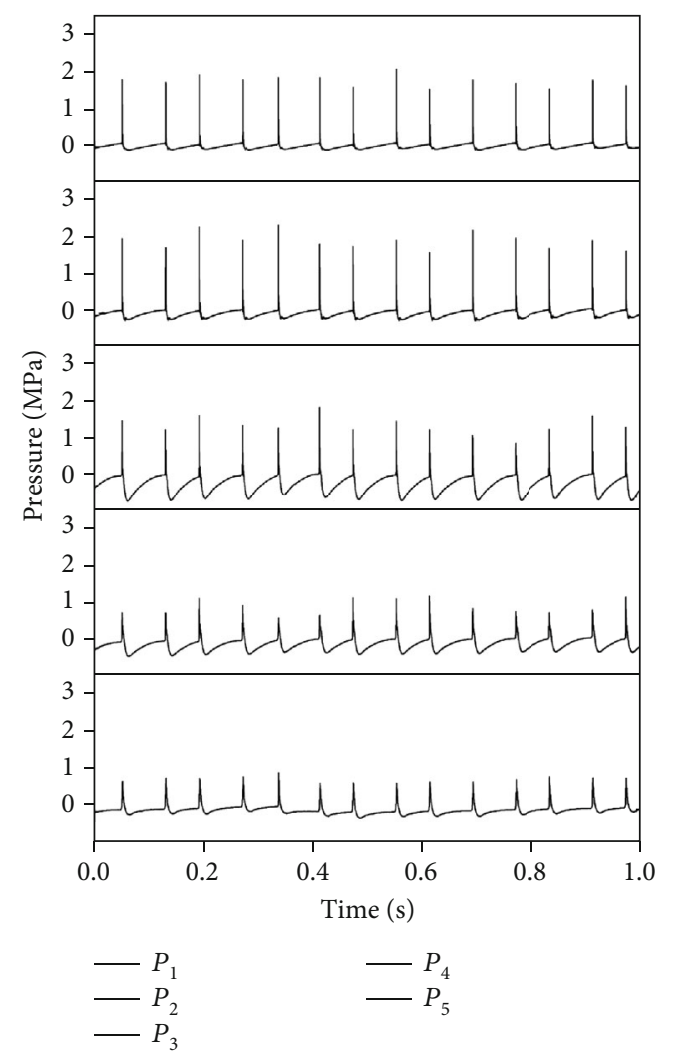

(a) Case 7

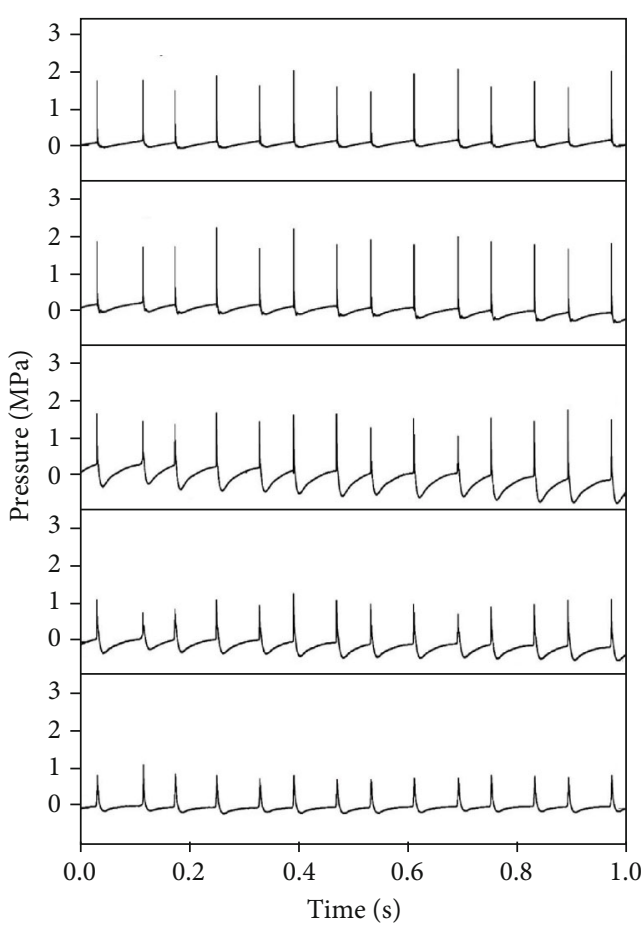

$\begin{array}{ll}-P_{1} & -P_{4} \\ -P_{2} & -P_{5} \\ -P_{3} & \end{array}$

(b) Case 8

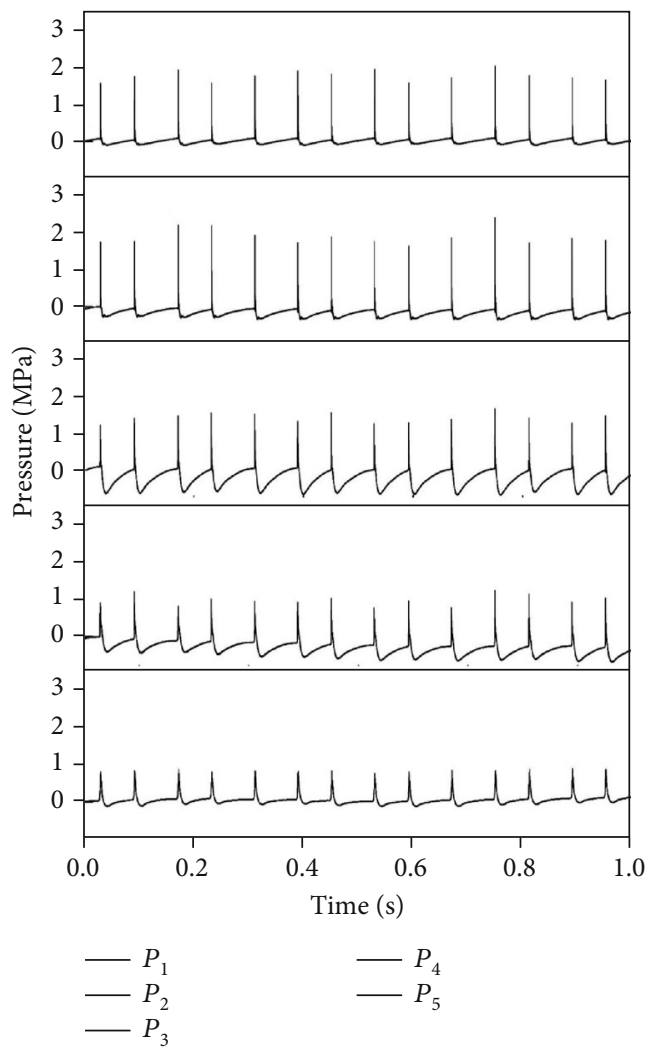

(c) Case 9

FIgURE 11: Pressure history for the reed valve. 


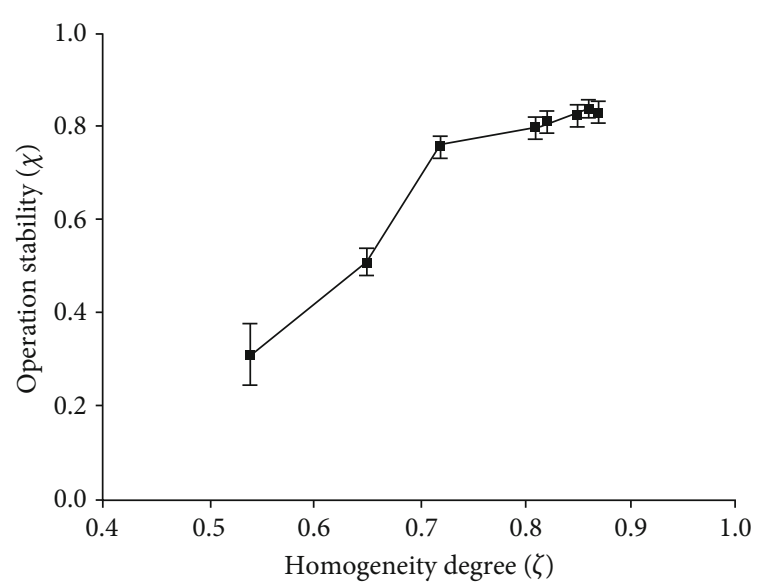

FIGURE 12: Operation stability of multicycle PDE for different homogeneity degrees.

Furthermore, compared with Figure 10, the detonation waves are achieved ahead of the $\mathrm{P} 4$ position in Figure 11. The DDT distance of PDE with the reed valve is shorter than that with the pore plate.

4.2. Operation Stability of PDE. To further study the relation between operation stability of PDE and homogeneity degree of two-phase mixture, the operation stability is ascertained by the statistical analysis of peak pressure at $P_{5}$. It can be calculated using the following equation:

$$
\chi=1-\frac{S_{p}^{2}}{\bar{p}^{2}}=1-\frac{1 /(N-1) \sum_{i=1}^{N}\left(p_{i}-\bar{p}\right)^{2}}{\bar{p}^{2}},
$$

where $p_{\mathrm{i}}$ is the peak pressure of cycle $i$ at $P_{5}, N$ is the number of cycle used in the average, and $\bar{p}$ is the average of peak pressure. The closer the operation stability $\chi$ is to 1 , the more stable the multicyclic working of PDE is.

The operation stability of multicycle PDE $\chi$ for different homogeneity degree $\zeta$ is plotted in Figure 12. It can be seen from the figure that the operation stability of multicycle PDE increases when the homogeneity degree improves. The highest gained operation stability $\chi$ is 0.841 when $\zeta$ is 0.86 at case 9 . When homogeneity degree $\zeta$ is larger than $0.72, \zeta$ has a small impact on the operation stability of multicycle PDE, and the operation stability slightly increases with the increase of $\zeta$, whereas as $\zeta$ is lower than $0.72, \zeta$ has a significant influence and the operation stability rapidly declines with the decrease of $\zeta$. This is attributed to the ignition fails or detonation wave is not achieved in some cycles for the lower homogeneity degree. Therefore, the homogeneity degree $\zeta=0.72$ is a critical value of stable working of multicycle two-phase PDE. For the higher homogeneity degree, the operation stability $\chi$ is influenced by both the homogeneity degree and turbulence Therefore, the operation stability $\chi$ presents a small decline as $\zeta$ increases from 0.86 to 0.87 .

\section{Summary and Conclusions}

This work first quantitatively investigated the effect of the homogeneity degree of mixture on the operation stability of multicycle PDE by PIV with 9 mixing schemes. Based on the experimental results, it can be concluded as follows:

(1) Homogeneity degree, which takes fuel distribution with space and time into account, was proposed to quantitatively assess the mixture of liquid fuel and air. The homogeneity degree of axial inlet way was better than that of tangential and radial inlet way. Centrifugal forces produced from rotating airflow led to a spiral movement of fuel droplets along the tube wall, and just a few fuel droplets are present near the axis for tangential air inlet way without mixing reinforcement devices. The mixing reinforcement devices, such as pore plate and reed valve, not only improved fuel distribution near spark plug but also reduced the effect of inlet ways on the homogeneity degree. As for the arrangement, the reed valve performs better than the pore plate

(2) Operation stability of multicycle PDE was presented by the statistical analysis of peak pressure at the outlet of the detonation tube. The relationship between operation stability of PDE and homogeneity degree of mixture was quantitatively analyzed. The homogeneity degree of $\zeta=0.72$ was a critical value for stable working of multicycle two-phase PDE. When homogeneity degree $\zeta$ was lower than 0.72 , it had significant influences on the operation stability of multicycle PDE and detonation waves in some cycles were not achieved due to poor homogeneity degree. The homogeneity degree $\zeta$ could have a small impact on the operation stability of multicycle PDE if $\zeta$ was larger than 0.72 . Therefore, it was necessary to achieve a homogeneity degree $\zeta$ of above 0.72 to ensure PDE works steadily. These generated results were expected to improve the operation stability and to offer guidelines for the design of PDE's mixing scheme

(3) Since detonation wave is the complex of shock wave and flame, the stability of detonation wave is preliminarily discussed by using pressure wave time curve in this paper, which is not enough. The future research plan is that Schlieren technology will be used to specifically study the shock-flame structure under the condition of nonuniform mixed detonating mixture

\section{Nomenclature}

\section{$d: \quad$ Diameter}

DDT: Deflagration-to-detonation transition

$G_{i, t}$ : Gray value of pixel point $i$ at time $t$

$\bar{G}: \quad$ Average gray value

$I_{\text {spf }}: \quad$ Specific impulse based on fuel

$m$ : Total number of pictures 
$n: \quad$ Sum of pixel points

$N$ : Number of operation cycle

$P_{i}: \quad$ Mounted position of transducers $i$

$p_{i}: \quad$ Peak pressure of cycle $i$ at $P_{5}$

$\bar{p}$ : $\quad$ Average of peak pressure

PDE: Pulse detonation engine

$\alpha$ : $\quad$ Maximum opening angle of valve sheet

$\zeta: \quad$ Homogeneity degree of fuel distribution

$\chi$ : Operation stability of PDE.

\section{Data Availability}

The data is all in the article. There is no additional data.

\section{Conflicts of Interest}

The authors declare no competing financial interest.

\section{Acknowledgments}

This research was supported by the National Natural Science Foundation of China through Grant No. 51606100, the Natural Science Foundation of Jiangsu Province, China, through Grant No. BK20150782, and the Fundamental Research Funds for the Central Universities though Grant Nos. 30915118836 and 309171B8806.

\section{References}

[1] V. F. Nikitin, V. R. Dushin, Y. G. Phylippov, and J. C. Legros, "Pulse detonation engines: technical approaches," Acta Astronautica, vol. 64, no. 2-3, pp. 281-287, 2009.

[2] J. E. Shepherd, "Detonation in gases," Proceedings of the Combustion Institute, vol. 32, no. 1, pp. 83-98, 2009.

[3] R. Driscoll, A. St George, D. Munday, and E. J. Gutmark, "Optimization of a multiple pulse detonation enginecrossover system," Applied Thermal Engineering, vol. 96, pp. 463-472, 2016.

[4] M. Shimo and S. D. Heister, "Multicyclic-detonation-initiation studies in valveless pulsed detonation combustors," Journal of Propulsion and Power, vol. 24, no. 2, pp. 336-344, 2008.

[5] F. Y. Zhang, T. Fujiwara, T. Miyasaka et al., "Experimental study of key issues on pulse detonation engine development," Transactions of The Japan Society for Aeronautical and Space Sciences., vol. 45, no. 150, pp. 243-248, 2003.

[6] Z. Wang, Z. Liang, Y. Zhang, and L. Zheng, "Direct-connected experimental investigation on a pulse detonation engine," Proceedings of the Institution of Mechanical Engineers, Part G: Journal of Aerospace Engineering, vol. 231, no. 7, pp. 1338-1346, 2016.

[7] Y. Huang, H. Tang, J. Li, and J. Wang, "Deflagration to detonation transition of kerosene-air mixtures in a small-scale pulse detonation engine," Proceedings of the Institution of Mechanical Engineers Part G: Journal of Aerospace Engineering, vol. 225, no. 4, pp. 441-448, 2011.

[8] E. K. Dabora, K. W. Ragland, and J. A. Nicholls, "Drop-size effects in spray detonations," Symposium (International) on Combustion, vol. 12, no. 1, pp. 19-26, 1969.

[9] Z. C. Lin, J. A. Nicholls, M. J. Tang, C. W. Kauffman, and M. Sichel, "Vapor pressure and sensitization effects in detona- tion of a decane spray," Symposium (International) on Combustion, vol. 20, no. 1, pp. 1709-1716, 1985.

[10] S. Cheatham and K. Kailasanath, "Single-cycle performance of idealized liquid-fueled pulse detonation engines," AIAA Journal, vol. 43, no. 6, pp. 1276-1283, 2005.

[11] S. Cheatham and K. Kailasanath, "Numerical modelling of liquid-fuelled detonations in tubes," Combustion Theory and Modelling, vol. 9, no. 1, pp. 23-48, 2005.

[12] V. Tangirala, A. Dean, O. Peroomian, and S. Palaniswamy, "Investigations of two-phase detonations for performance estimations of a pulse detonation engine," in 45th AIAA Aerospace Sciences Meeting and Exhibit, p. 1173, Reno, NV, 2007.

[13] J. Lasheras, B. Varatharajan, C. Varga, and F. Williams, "Studies of fuel distribution and detonation chemistry for pulse detonation engines," in ISOABE, ISABE- International Symposium on Air Breathing Engines, 15th, p. 1174, Bangalore, India, 2001.

[14] Z. Wang, C. Yan, W. Fan, and L. Zheng, "Experimental study of atomization effects on two-phase pulse detonation engines," Proceedings of the Institution of Mechanical Engineers Part G: Journal of Aerospace Engineering, vol. 223, no. 6, pp. 721728, 2009.

[15] C. Tucker, P. King, R. Bradley, and F. Schauer, "The use of a flash vaporization system with liquid hydrocarbon fuels in a pulse detonation engine," in 42nd AIAA Aerospace Sciences Meeting and Exhibit, p. 0868, Reno, Nevada, 2004.

[16] K. C. Tucker, P. I. King, and F. R. Schauer, "Hydrocarbon fuel flash vaporization for pulsed detonation combustion," Journal of Propulsion and Power, vol. 24, no. 4, pp. 788796, 2008.

[17] C. Miser, P. King, and F. Schauer, "PDE flash vaporization system for hydrocarbon fuel using thrust tube waste heat," in 41st AIAA/ASME/SAE/ASEE Joint Propulsion Conference \& Exhibit, p. 3511, Tucson, Arizona, 2005.

[18] T. M. Helfrich, P. I. King, J. L. Hoke, and F. R. Schauer, "Effect of supercritical fuel injection on cycle performance of pulsed detonation engine," Journal of Propulsion and Power, vol. 23, no. 4, pp. 748-755, 2007.

[19] Z. C. Fan, W. Fan, H. Tu, J. L. Li, and C. J. Yan, "The effect of fuel pretreatment on performance of pulse detonation rocket engines," Experimental Thermal and Fluid Science, vol. 41, pp. 130-142, 2012.

[20] E. Barbour, L. Ma, J. Jeffries, R. Hanson, C. Brophy, and J. Sinibaldi, "Real-time measurements of $\mathrm{C} 2 \mathrm{H} 4$ concentration with application to PDEs operating on oxygen and air," in 41st AIAA/ASME/SAE/ASEE Joint Propulsion Conference \& Exhibit, p. 4376, Tucson, Arizona, 2005.

[21] L. Ma, J. Jeffries, R. Hanson, K. Hinckley, P. Pinard, and A. Dean, "Characterization of the fuel fill process in a multicycle pulse detonation engine using a diode-laser sensor," in 41st AIAA/ASME/SAE/ASEE Joint Propulsion Conference \& Exhibit, p. 3834, Tucson, AZ, 2005.

[22] C. M. Brophy and R. K. Hanson, "Fuel distribution effects on pulse detonation engine operation and performance," Journal of Propulsion and Power, vol. 22, no. 6, pp. 11551161, 2006.

[23] C. M. Brophy, J. O. Sinibaldi, and L. Ma, "Effects of nonuniform mixture distributions on pulse detonation engine performance," in 43rd AIAA Aerospace Sciences Meeting and Exhibit, p. 1304, Reno, Nevada, 2005. 
[24] H. D. Perkins and C. J. Sung, "Effects of fuel distribution on detonation tube Performance," Journal of Propulsion and Power, vol. 21, no. 3, pp. 539-545, 2005.

[25] M. K. Brett, Spray Detonation in a Well-Characterized Homogeneous Mixture, Stanford University, Stanford, California, 2003.

[26] O. Peraldi, R. Knystautas, and J. H. Lee, "Criteria for transition to detonation in tubes," Symposium (International) on Combustion, vol. 21, no. 1, pp. 1629-1637, 1988. 